DPNU 00-11

\title{
Vacuum Polarization of Massive Scalar Fields on the Black Hole Horizon
}

\author{
Hiroko Koyama, Yasusada Nambu and Akira Tomimatsu \\ Department of Physics, Graduate School of Science, Nagoya University, Nagoya 464-8602, Japan
}

(November 21, 2018)

\begin{abstract}
Vacuum polarization of massive scalar fields in a thermal state at arbitrary temperature is studied near the horizon of a Reissner-Nordström black hole. We derived an analytic form of $\left\langle\phi^{2}\right\rangle$ approximately in the large mass limit near the black hole horizon. We uses the zeroth order WKB approximation and power series expansion near the horizon for the Euclideanized mode function. Our formula for the vacuum polarization shows regular behavior on the horizon if the temperature of the scalar field is equal to the Hawking temperature of the black hole. The finite part of the vacuum polarization agrees with the result of the DeWitt-Schwinger approximation up to $O\left(\mathrm{~m}^{-4}\right)$ which is the next leading order of the expansion.
\end{abstract}

PACS number(s) 04.62.+v, 04.70.Dy 


\section{INTRODUCTION}

Since Hawking's discovery of black holes radiation [四], it has been an important subject to investigate quantum effects in black hole spacetimes and a great deal of effort has been done. The expectation values of the regularized vacuum polarization $\left\langle\phi^{2}\right\rangle$ and the stress energy tensor $\left\langle T_{\mu \nu}\right\rangle$ are important quantities to understand quantum effects in black hole spacetimes. They represent the effect of quantum fluctuations and back reaction on black hole spacetimes and play an important role in the context of semi-classical theory of quantum gravity.

For a scalar field in the large mass limit, of which Compton length is much smaller than the characteristic curvature scale of background geometry, we can evaluate regularized $\left\langle\phi^{2}\right\rangle$ and $\left\langle T_{\mu \nu}\right\rangle$ by using the DeWitt-Schwinger approximation [2 6]. This is an adiabatic expansion in terms of inverse power series of the mass of scalar field. One can evaluate divergent and finite parts of $\left\langle\phi^{2}\right\rangle$ and $\left\langle T_{\mu \nu}\right\rangle$ up to arbitrary order. Frolov and Zelnikov [4, 5] suggest that the finite parts of $\left\langle\phi^{2}\right\rangle$ and $\left\langle T_{\mu \nu}\right\rangle$ calculated by the DeWitt-Schwinger expansion coincides with the value calculated by assuming the Hartle-Hawking state [7] for the scalar field, although the DeWitt-Schwinger expansion does not impose any condition on the state of the scalar field. The justification is confirmed numerically near the horizon [8,9].

The Hartle-Hawking state is defined by requiring that the Euclidean Green's function is regular on the horizon [7]. Its physical meaning is that a black hole is placed in a cavity and is equilibrium with thermal black-body radiation. The thermal state is defined by the Euclidean Green's function by imposing periodicity in the imaginary time coordinate with a period of the inverse of the temperature. On the order hand, in the DeWitt-Schwinger expansion, the condition which characterizes the thermal state is not imposed. So it is not obvious whether the DeWitt-Schwinger expansion yields the same finite part of the Hartle-Hawking state near the horizon.

In this paper, we consider scalar fields in static spherically symmetric background spacetimes. By assuming that the mass of scalar fields is large, we calculate $\left\langle\phi^{2}\right\rangle$ in the thermal state with arbitrary temperature using point splitting method near the horizon. A similar calculation is already done by Anderson et al. [8,9, 9, but their expression of the vacuum polarization diverges on the horizon which contradicts with their numerical calculation. Our purpose is to derive the vacuum polarization which is regular on the horizon, and resolve why the finite part of $\left\langle\phi^{2}\right\rangle$ in the Hartle-Hawking state near the horizon can be approximated by the DeWitt-Schwinger expansion which does not assume any condition on the thermal state.

The plan of this paper is as follows. In Sec.II, we review the method to calculate $\left\langle\phi^{2}\right\rangle$ in a thermal state using point splitting regularization. In Sec.III, we present our method

to calculate $\left\langle\phi^{2}\right\rangle$ in the large mass limit near the black hole horizon. Sec.IV is devoted to conclusion and discussion. Throughout the paper, we use units such that $\hbar=c=G=k_{B}=$ 1. Our sign conventions are those of Misner, Thorne and Wheeler [10].

\section{DERIVATION OF VACUUM POLARIZATION $\left\langle\phi^{2}\right\rangle$ IN A THERMAL STATE}

We consider a minimally coupled scalar field with mass $m$ in a thermal state at arbitrary temperature $T$ in a Reissner-Nordström spacetime. The metric in Euclidean section $\tau=-i t$ 
is given by

$$
d s^{2}=f d \tau^{2}+\frac{1}{f} d r^{2}+r^{2} d \Omega^{2},
$$

where $f=\left(r-r_{+}\right)\left(r-r_{-}\right) / r^{2}, r_{+}$and $r_{-}$are the location of inner and outer horizon, respectively. The surface gravity of a black hole is given by $\kappa=\left(r_{+}-r_{-}\right) /\left(2 r_{+}^{2}\right)$. The vacuum polarization $\left\langle\phi^{2}\right\rangle$ can be computed from the Euclidean Green's function by noting the identity

$$
\left\langle\phi^{2}\right\rangle_{\mathrm{unreg}}=\frac{1}{2} G^{(1)}(x, x)=i G_{F}(x, x)=G_{E}(x, x),
$$

where $G^{(1)}$ is the Hadamard Green's function, $G_{F}$ is the Feynman Green's function, and $G_{E}$ is the Euclidean Green's function, respectively. $G_{E}$ is divergent at coincident limit and must be regularized. In this paper, the covariant point-splitting method is employed for the regularization of ultraviolet divergences. We start from an expression for $G_{E}\left(x, x^{\prime}\right)$ when the points $x$ and $x^{\prime}$ are separated. Next we prepare an appropriate counterterm to subtract the divergence, and then take coincident limit. This procedure is shown schematically as

$$
\left\langle\phi^{2}\right\rangle_{\mathrm{reg}}=\lim _{x^{\prime} \rightarrow x}\left[G_{E}\left(x, x^{\prime}\right)-G_{D S}\left(x, x^{\prime}\right)\right]
$$

where $G_{D S}\left(x, x^{\prime}\right)$ is a point splitting counterterm needed to regularize $G_{E}\left(x, x^{\prime}\right)$. The Euclidean Green's function obeys the equation

$$
\left[\square-m^{2}\right] G_{E}\left(x, x^{\prime}\right)=-\frac{\delta^{4}\left(x, x^{\prime}\right)}{g^{1 / 2}(x)},
$$

where $m$ is the mass of the scalar field and d'Alembertian $\square$ evaluated using the Euclidean metric (1). The point splitting counter term needed to renormalize $G_{E}\left(x, x^{\prime}\right)$ in arbitrary spacetime is [2]

$$
\begin{aligned}
G_{D S}\left(x, x^{\prime}\right)= & \frac{1}{8 \pi^{2} \sigma}+\frac{m^{2}+\left(\xi-\frac{1}{6}\right) R}{8 \pi^{2}}\left[\gamma+\frac{1}{2} \ln \left[\frac{m^{2} \sigma}{2}\right]\right] \\
& -\frac{m^{2}}{16 \pi^{2}}+\frac{1}{96 \pi^{2}} R_{\alpha \beta} \frac{\sigma^{, \alpha} \sigma^{, \beta}}{\sigma},
\end{aligned}
$$

where $\xi$ is coupling constant to the scalar curvature $R, \sigma$ is the one half the square of geodesic distance between the points $x$ and $x^{\prime}$ and $\gamma$ is Euler's constant.

For simplicity of calculation, the quantity $\left\langle\phi^{2}\right\rangle$ is evaluated by timelike point separation $\tau^{\prime} \neq \tau$. By assuming that the Green's function is periodic in $\epsilon=\tau-\tau^{\prime}$ with period $1 / T$, the expression for the vacuum polarization in the thermal state at temperature $T$ is given by

$$
\begin{aligned}
&\left\langle\phi^{2}\right\rangle_{T}= \lim _{\tau^{\prime} \rightarrow \tau}\left[G_{E}\left(x, \tau ; x, \tau^{\prime}\right)-G_{D S}\left(x, \tau ; x, \tau^{\prime}\right)\right] \\
&= \lim _{\tau^{\prime} \rightarrow \tau}\left[\frac{T}{4 \pi} \sum_{l=0}^{\infty}\left[(2 l+1) p_{0 l} q_{0 l}-\frac{1}{r \sqrt{f}}\right]\right. \\
&\left.\quad+\frac{T}{2 \pi} \sum_{n=1}^{\infty} \cos \left[n 2 \pi T\left(\tau-\tau^{\prime}\right)\right] \sum_{l=0}^{\infty}\left[(2 l+1) p_{n l} q_{n l}-\frac{1}{r \sqrt{f}}\right]-G_{D S}\left(x, \tau ; x, \tau^{\prime}\right)\right] \\
& \equiv\left\langle\phi^{2}\right\rangle_{n=0}+\left\langle\phi^{2}\right\rangle_{n \geq 1}-\left\langle\phi^{2}\right\rangle_{D S},
\end{aligned}
$$


where

$$
\left\langle\phi^{2}\right\rangle_{D S}=\frac{m^{2}}{8 \pi^{2}}\left(\ln \frac{m \sqrt{f} \epsilon}{2}+\gamma-\frac{1}{2}\right)-\frac{1}{4 \pi^{2} f \epsilon^{2}}+\left(\frac{f^{\prime 2}}{192 \pi^{2} f}-\frac{f^{\prime \prime}}{96 \pi^{2}}-\frac{f^{\prime}}{48 \pi^{2} r}\right) .
$$

The term $1 /(r \sqrt{f})$ is subtracted to cancel the superficial divergence which comes from the choice of timelike point-splitting. $p_{n l}$ and $q_{n l}$ are independent solutions of the mode equation

$$
\frac{d^{2} \chi_{n l}}{d r^{2}}+\left[\frac{2}{r}+\frac{1}{f} \frac{d f}{d r}\right] \frac{d \chi_{n l}}{d r}-\left[\frac{(2 \pi n T)^{2}}{f^{2}}+\frac{l(l+1)}{r^{2} f}+\frac{m^{2}}{f}\right] \chi_{n l}=0
$$

and satisfy the Wronskian condition

$$
p_{n l} \frac{d q_{n l}}{d r}-q_{n l} \frac{d p_{n l}}{d r}=-\frac{1}{r^{2} f} .
$$

Anderson et al. 8,9] calculated the second and the third term of (6) analytically using the second order WKB approximation . Their result is

$$
\begin{aligned}
\left\langle\phi^{2}\right\rangle_{n \geq 1}-\left\langle\phi^{2}\right\rangle_{D S}=- & \frac{m^{2}}{16 \pi^{2}} \ln \left(\frac{m^{2} f}{16 \pi^{2} T^{2}}\right)+\frac{m^{2}}{16 \pi^{2}} \\
& +\frac{T^{2}}{12 f}-\frac{f^{\prime 2}}{192 \pi^{2} f}-\frac{f^{\prime \prime}}{96 \pi^{2}}-\frac{f^{\prime}}{48 \pi^{2} r} .
\end{aligned}
$$

The expression (10) was obtained by taking into account only the contribution from $n \geq 1$ mode. In the case of massless scalar fields, excluding the lowest frequency mode $n=0$ corresponds to imposing an infrared cutoff. The expression (10) gives good approximation for massless fields both near and far from the horizon in Schwarzschild and Reissner-Nordström spacetimes. This was confirmed by their numerical calculation [9].

When the result (10) is applied to the massive scalar field, however, two unpleasant features appear. Firstly, the regularized expression always has logarithmic divergence on the horizon $f=0$ even if the scalar field is in the Hartle-Hawking state. It is not consistent with their numerical work which shows regular behavior of $\left\langle\phi^{2}\right\rangle_{T}$ on the horizon. Secondly, the finite terms of $O\left(\mathrm{~m}^{2}\right)$ and $O\left(\mathrm{~m}^{0}\right)$ are left in the regularized expression. This disagrees with the result of the DeWitt-Schwinger approximation by Frolov [4 which does not contain such finite terms. Therefore the expression (10) cannot yield good approximation for the massive scalar field. In the next section, we present the method to improve their approximations and resolve these problems.

\section{VACUUM POLARIZATION NEAR THE HORIZON IN THE LARGE MASS LIMIT}

In this section, we present the method to resolve the unwanted behavior arose in the expression of $\left\langle\phi^{2}\right\rangle_{T}$ near the horizon $f \sim 0$. We assume that the mass $m$ of the scalar field is large:

$$
m \gg \frac{1}{r_{+}} .
$$

We evaluate $n \geq 1$ and $n=0$ contribution to $\left\langle\phi^{2}\right\rangle_{T}$ separately. 


\section{A. $n \geq 1$ contribution}

We can use the WKB approximation for $n \geq 1$ mode. The zeroth order WKB solution is given by

$$
\begin{gathered}
p_{n}=\frac{1}{\sqrt{2 r^{2} W}} \exp \left[\int d r \frac{W}{f}\right], \quad q_{n}=\frac{1}{\sqrt{2 r^{2} W}} \exp \left[-\int d r \frac{W}{f}\right], \\
; \quad W^{2}=\kappa^{2} n^{2}+m^{2} f+\frac{\left(l+\frac{1}{2}\right)^{2}}{r^{2}} f=\left(\alpha_{n}^{2}+\frac{\left(l+\frac{1}{2}\right)^{2}}{r^{2}}\right) f \\
\alpha_{n}^{2}=m^{2}+\frac{\kappa^{2} n^{2}}{f}, \quad \kappa=2 \pi T .
\end{gathered}
$$

The WKB approximation is correct near the horizon. Using this solution, we have

$$
\left\langle\phi^{2}\right\rangle_{n \geq 1}=\frac{T}{2 \pi} \sum_{n=1}^{\infty} \cos (n \kappa \epsilon) I_{n}
$$

where

$$
\begin{aligned}
I_{n} & \equiv \sum_{l=0}^{\infty}\left[(2 l+1) p_{n} q_{n}-\frac{1}{r \sqrt{f}}\right] \\
& =\frac{1}{r \sqrt{f}} \sum_{l=0}^{\infty}\left\{\frac{l+\frac{1}{2}}{\left[r^{2} \alpha_{n}^{2}+\left(l+\frac{1}{2}\right)^{2}\right]^{1 / 2}}-1\right\} .
\end{aligned}
$$

$I_{n}$ can be evaluated using the Plana sum formula [8,9] under the assumption $f \sim 0, \alpha_{n} \sim$ $\kappa n / f$ :

$$
\begin{aligned}
\sum_{l=0}^{\infty} \frac{l+\frac{1}{2}}{\left[r^{2} \alpha_{n}^{2}+\left(l+\frac{1}{2}\right)\right]^{1 / 2}=} & \frac{1}{2} \frac{\frac{1}{2}}{\left[r^{2} \alpha_{n}^{2}+\frac{1}{4}\right]^{1 / 2}}+\int_{0}^{\infty} d l \frac{l+\frac{1}{2}}{\left[r^{2} \alpha_{n}^{2}+\left(l+\frac{1}{2}\right)^{2}\right]^{1 / 2}} \\
& +i \int_{0}^{\infty} \frac{d l}{e^{2 \pi l}-1}\left\{\frac{i l+\frac{1}{2}}{\left[r^{2} \alpha_{n}^{2}+\left(i l+\frac{1}{2}\right)^{2}\right]^{1 / 2}}-(l \rightarrow-l)\right\} \\
= & {\left[\left(l+\frac{1}{2}\right)^{2}+r^{2} \alpha_{n}^{2}\right]_{l=0}^{1 / 2}+O\left(\frac{f}{r \kappa n}\right) . }
\end{aligned}
$$

Therefore, for $n \geq 1$,

$$
\begin{aligned}
I_{n} & =\frac{1}{r \sqrt{f}}\left\{-\left[\frac{1}{4}+r^{2}\left(m^{2}+\frac{r^{2} \kappa^{2} n^{2}}{f^{2}}\right)\right]^{1 / 2}+O\left(\frac{f}{r \kappa n}\right)\right\} \\
& =-\frac{\kappa n}{f}-\frac{m^{2}}{2 \kappa n}+O\left(\frac{f}{r n^{3}}, \frac{\sqrt{f}}{r^{2} \kappa n}\right) .
\end{aligned}
$$


By taking $n$ sum, $O\left(f /\left(r n^{3}\right), \sqrt{f} /\left(r^{2} \kappa n\right)\right)$ terms give finite values which becomes zero on the horizon and we can omit these terms. Using the formula

$$
\begin{aligned}
\kappa \sum_{n=1}^{\infty} \frac{\cos (n \kappa \epsilon)}{n \kappa} & =-\frac{1}{2} \ln \left(\kappa^{2} \epsilon^{2}\right)+O\left(\epsilon^{2}\right), \\
\kappa \sum_{n=1}^{\infty} n \kappa \cos (n \kappa \epsilon) & =-\frac{1}{\epsilon^{2}}-\frac{\kappa^{2}}{12}+O\left(\epsilon^{2}\right),
\end{aligned}
$$

we have

$$
\begin{aligned}
\left\langle\phi^{2}\right\rangle_{n \geq 1} & \approx-\frac{\kappa^{2}}{4 \pi^{2} f} \sum_{n=1}^{\infty} n \cos (n \kappa \epsilon)-\frac{m^{2}}{8 \pi^{2}} \sum_{n=1}^{\infty} \frac{\cos (n \kappa \epsilon)}{n} \\
& =\frac{1}{4 \pi^{2} f \epsilon^{2}}+\frac{\kappa^{2}}{48 \pi^{2} f}+\frac{m^{2}}{16 \pi^{2}} \ln \left(k a p p a^{2} \epsilon^{2}\right) .
\end{aligned}
$$

Using the DeWitt-Schwinger counter term (『), the regularized expression of the expectation value becomes

$$
\left\langle\phi^{2}\right\rangle_{n \geq 1}-\left\langle\phi^{2}\right\rangle_{D S}=-\frac{m^{2}}{16 \pi^{2}} \ln \left(\frac{m^{2} f}{16 \pi^{2} T^{2}}\right)+\frac{m^{2}}{16 \pi^{2}}+\frac{T^{2}}{12 f}-\frac{f^{\prime 2}}{192 \pi^{2} f}-\frac{f^{\prime \prime}}{96 \pi^{2}}-\frac{f^{\prime}}{48 \pi^{2} r} .
$$

This gives the same expression of Anderson et al. [9], who used the second order WKB solution. Near the black hole horizon, it is sufficient to use the zeroth order WKB solution to reproduce the result of the second order WKB approximation. On the horizon $f=0$, this expression diverge and we must examine the contribution from $n=0$ mode.

\section{B. $n=0$ contribution}

We must recall that the expression (10) does not include $n=0$ mode but there is no reason to exclude this contribution in the case of the massive scalar field. So we must investigate the contribution of $n=0$ mode. Since the WKB method breaks down for $n=0$ mode near the horizon, we cannot apply the approximation used by Anderson et al. [9]. We solve $n=0$ mode function by the following method.

As we assume that the mass of the scalar field is large, a dimensionless constant

$$
\epsilon \equiv \frac{r_{+}-r_{-}}{4 r_{+}\left[m^{2} r_{+}^{2}+l(l+1)\right]}
$$

becomes smaller than unity for all value of $l$ :

$$
\epsilon \ll 1 \quad \text { (for all } l \text { ) }
$$

We rescale the radial coordinate $r$ as follows:

$$
x \equiv \sqrt{\frac{r-r_{+}}{\epsilon r_{+}}} .
$$


Assuming that $x$ is $O(1)$ quantity, this rescaling of the radial coordinate means we are concentrate on the region $\left(r-r_{+}\right) \sim O(\epsilon)$, which is the region near the black hole horizon. Using $x$ and $\epsilon$, the radial equation (8) for $n=0$ mode can be written as

$$
\frac{d^{2} \chi_{0 l}}{d x^{2}}+\left[\frac{1}{x}+F(x, \epsilon)\right] \frac{d \chi_{0 l}}{d x}-[1+G(x, \epsilon)] \chi_{0 l}=0,
$$

where

$$
\begin{aligned}
& F(x, \epsilon)=\frac{2 \epsilon x}{2 \kappa r_{+}+\epsilon x^{2}} \\
& G(x, \epsilon)=\frac{16 \epsilon}{2 \kappa r_{+}+\epsilon x^{2}}\left[\kappa r_{+}+2 m^{2} r_{+}^{2}\left(2 \epsilon^{2} x^{2}+3 \epsilon^{3} x^{4}\right)\right] .
\end{aligned}
$$

We can express the approximate solution of the mode equation (23) by the power series expansion with respect to a small parameter $\epsilon$ :

$$
\chi_{0 l}(x, \eta)=\chi_{0 l}^{(0)}(x)+\epsilon \chi_{0 l}^{(1)}(x)+\epsilon^{2} \chi_{0 l}^{(2)}(x)+\cdots .
$$

$\chi_{0 l}^{(n)}$ obeys

$$
\begin{aligned}
\frac{d \chi_{0 l}^{(0)}}{d x^{2}}+ & \frac{1}{x} \frac{d \chi_{0 l}^{(0)}}{d x}-\chi_{0 l}^{(0)}=0 \\
\frac{d \chi_{0}^{(n)}}{d x^{2}}+\frac{1}{x} \frac{d \chi_{0 l}^{(n)}}{d x}-\chi_{0 l}^{(n)}= & \\
& \sum_{k=1}^{n} \frac{1}{k !}\left[-\left.\frac{\partial^{k} F}{\partial \epsilon^{k}}\right|_{\epsilon=0} \frac{d \chi_{0 l}^{(n-k)}}{d x}+\left.\frac{\partial^{k} G}{\partial \epsilon^{k}}\right|_{\epsilon=0} \chi_{0 l}^{(n-k)}\right] \quad(\text { for } n \geq 1)
\end{aligned}
$$

We can obtain solutions of these equations by using modified Bessel functions. For example, the lowest order solution is given by

$$
p_{l 0}^{(0)}=I_{0}(x), \quad q_{l 0}^{(0)}=\frac{2}{r_{+}-r_{-}} K_{0}(x) .
$$

Substituting the expansion (26) to

$$
\left\langle\phi^{2}\right\rangle_{n=0}=\frac{T}{4 \pi} \sum_{l=0}^{\infty}\left[(2 l+1) p_{l 0} q_{l 0}-\frac{1}{r \sqrt{f}}\right]
$$

and taking summation about $l$ using the Plana sum formula, $\left\langle\phi^{2}\right\rangle_{n=0}$ is written as the power series expansion with respect to $\left(m r_{+}\right)^{-1}$. We calculated $\left\langle\phi^{2}\right\rangle_{n=0}$ up to $O\left(\left(m r_{+}\right)^{-4}\right)$, which is accomplished by the $\epsilon$ expansion of the mode function up to $O\left(\epsilon^{3}\right)$. By taking into the account of the contribution of $n=0$ mode, we finally get the form of the vacuum polarization 
near the horizon as follows:

$$
\begin{aligned}
\left\langle\phi^{2}\right\rangle_{T} & =\left(T-\frac{\kappa}{2 \pi}\right)\left\{-\frac{m^{2}}{16 \pi \kappa}+\frac{m^{2}}{8 \pi \kappa}\left[2 \gamma+\ln \frac{m^{2}\left(r-r_{+}\right)}{2 \pi T}\right]-\frac{1}{12 \pi r_{+}}\right\} \\
& +\left\{T^{2}-\left(\frac{\kappa}{2 \pi}\right)^{2}\right\}\left[\frac{1}{24 \kappa\left(r-r_{+}\right)}-\frac{r_{-}}{48 r_{+}^{3} \kappa^{2}}\right] \\
& +\frac{m^{2}}{16 \pi^{2} \kappa}(\kappa+2 \pi T) \ln \left(\frac{2 \pi T}{\kappa}\right) \\
& +\frac{T}{4 \pi} \frac{1}{45 m^{2}} \frac{1}{\left(r_{+}-r_{-}\right) r_{+}^{2}}\left[3-\frac{6 r_{-}}{r_{+}}+\frac{4 r_{-}^{2}}{r_{+}^{2}}\right] \\
& +\frac{T}{4 \pi} \frac{2}{315 m^{4}} \frac{1}{\left(r_{+}-r_{-}\right) r_{+}^{4}}\left[-10+39 \frac{r_{-}}{r_{+}}-52 \frac{r_{-}^{2}}{r_{+}^{2}}+23 \frac{r_{-}^{3}}{r_{+}^{3}}\right]+O\left(\left(m r_{+}\right)^{-6}\right) .
\end{aligned}
$$

The divergences in $\left\langle\phi^{2}\right\rangle_{T}$ appears only in the terms of $O\left(m^{2}\right)$ and $O\left(m^{0}\right)$. Taking $f \rightarrow 0$ limit, $O\left(m^{2}\right)$ term diverge as $\ln \left(r-r_{+}\right)$and $O\left(m^{0}\right)$ as $\left(r-r_{+}\right)^{-1}$. These divergence disappear completely if the temperature of the scalar field equals $T=\kappa / 2 \pi \equiv T_{H}$, where $T_{H}$ is the Hawking temperature. Specifying $T=T_{H}$ is equivalent to require the regurality of $\left\langle\phi^{2}\right\rangle$ on the horizon. In this case, the black hole is equilibrium with the thermal scalar field with temperature $T=T_{H}$. This is nothing but a Hartle-Hawking state.

We can evaluate a finite part of $\left\langle\phi^{2}\right\rangle_{T}$ in the Hartle-Hawking state on the horizon. The leading order of $\left\langle\phi^{2}\right\rangle_{T}$ at $T=T_{H}$ is $O\left(\mathrm{~m}^{-2}\right)$ because not only divergent but also finite terms contained in $O\left(\mathrm{~m}^{2}\right)$ and $O\left(\mathrm{~m}^{0}\right)$ terms vanish. The result is given by

$$
\begin{aligned}
\left\langle\phi^{2}\right\rangle_{T=T_{H}} & =\frac{1}{720 \pi^{2} m^{2}} \frac{1}{r_{+}^{4}}\left[3-\frac{6 r_{-}}{r_{+}}+\frac{4 r_{-}^{2}}{r_{+}^{2}}\right] \\
& +\frac{1}{2520 \pi^{2} m^{4}} \frac{1}{r_{+}^{6}}\left[-10+39 \frac{r_{-}}{r_{+}}-52 \frac{r_{-}^{2}}{r_{+}^{2}}+23 \frac{r_{-}^{3}}{r_{+}^{3}}\right]+O\left(m^{-6}\right) .
\end{aligned}
$$

This expression agrees with the result of the DeWitt-Schwinger expansion [4,6,8].

\section{CONCLUSION AND DISCUSSION}

We calculated $\left\langle\phi^{2}\right\rangle_{T}$ of a massive scalar field near the horizon of a Reissner-Nordström black hole using point splitting method. Our results are as follows: (i)all divergent and finite terms of $O\left(\mathrm{~m}^{2}\right)$ and $O\left(\mathrm{~m}^{0}\right)$ in $\left\langle\phi^{2}\right\rangle_{T}$ are canceled to be zero if the temperature of the scalar field equals to the Hawking temperature $T_{H}$ of a black hole. (ii)at this temperature, the leading order of the regularized $\left\langle\phi^{2}\right\rangle_{T}$ becomes $O\left(\mathrm{~m}^{-2}\right)$ and its value on the horizon agrees with that of the DeWitt-Schwinger expansion up to $O\left(\mathrm{~m}^{-4}\right)$. (i) and (ii) indicate that regularized $\left\langle\phi^{2}\right\rangle_{T}$ in the Hartle-Hawking state near the black hole horizon is well approximated by the DeWitt-Schwinger expansion. This confirms the results of [1, 5].

It is not trivial that the finite part of $\left\langle\phi^{2}\right\rangle_{T}$ in the thermal state at $T=T_{H}$ is reproduced by the DeWitt-Schwinger expansion which does not specify any thermal state of the scalar

field. It was shown that the leading order of the DeWitt-Schwinger expansion reproduces the value $\left\langle\phi^{2}\right\rangle_{T=0}$ of scalar field with large mass in general static spherical symmetric spacetime 
[11]. This result is natural because the scalar field with $T=0$ is in a vacuum state and the DeWitt-Schwinger expansion does not incorporate information on a thermal state.

Discrepancy between $\left\langle\phi^{2}\right\rangle_{T}$ in the Hartle-Hawking state and that in he DeWitt-Schwinger expansion will appear far from the horizon, because the thermal effect dominates the gravitational effect in the asymptotic flat region. If the thermal effect is dominant or comparable to the contribution from curvature effect near the horizon, there would also be discrepancy between $\left\langle\phi^{2}\right\rangle_{T}$ in the Hartle-Hawking state and that of the DeWitt-Schwinger expansion. However this is not seen from our results. This implies that the finite part of $\left\langle\phi^{2}\right\rangle_{T}$ in the Hartle-Hawking state near the black hole horizon is dominated by the contribution from curvature effect. Why the contribution from thermal effects to finite parts can be negligible? The characteristic scale for thermal state is the inverse of the temperature $1 / T_{H} \sim M$ which is a scale length of thermal fluctuation. On the other hand, the Compton length of the scalar field is $1 / m$. The contribution of the Hawking radiation to $\left\langle\phi^{2}\right\rangle$ is $\propto T_{H}^{4} / m^{2}$ and that of thermal excitation is $\propto \exp \left(-m / T_{H}\right)$. For $m \gg 1 / M$, the effect of thermal excitation is exponentially small. Furthermore, near the black hole horizon, almost all part of thermally excited particle is absorbed by the black hole. This is the reason of the DeWitt-Schwinger approximation gives the same result of Hartle-Hawking state on the horizon. 


\section{REFERENCES}

[1] S. W. Hawking, Commun. Math. Phys. 43, 199 (1975).

[2] S. M. Christensen, Phys. Rev. D 14, 2490 (1976).

[3] B. S. DeWitt, Phys. Rep. 19, 295 (1975).

[4] Frolov, Proceedings of Second Seminar on Quantum Gravity, Moscow, USSR (Plenum, New York, 1984), p. 303.

[5] V. P. Frolov and A. I. Zel'nikov, Phys. Lett. 115B, 372 (1982).

[6] L. Parker and D. J. Toms, Phys. Rev. D 31, 953 (1985).

[7] J. B. Hartle and S. W. Hawking, Phys. Rev. D 13, 2188 (1976).

[8] P. R. Anderson, Phys. Rev. D 41, 1152 (1990).

[9] P. R. Anderson, W. A. Hiscock, and D. A. Samuel, Phys. Rev. D 51, 4337 (1995).

[10] C. W. Misner, K. S. Thorne, and J. A. Wheeler, Gravitation (Freeman, San Francisco, 1973).

[11] R. Herman, Phys. Rev. D 58, 084028 (1998). 